\title{
APPROXIMATION BY CONTINUED FRACTIONS
}

\section{MELVYN B. NATHANSON}

ABSTRACT. Let $x$ be a real irrational number whose continued fraction has infinitely many partial quotients not less than $k$. A simple proof is given that the in equality $|x-p / q|<\left(k^{2}+4\right)^{-1 / 2} q^{-2}$ has infinitely many rational solutions $p / q$. The constant $\left(k^{2}+4\right)^{-1 / 2}$ is best possible.

Recently, J. H. E. Cohn [1] gave a simple proof of Hurwitz' theorem, that for any real irrational number $x$ there are infinitely many rational numbers $p / q$ such that $|x-p / q|<5^{-1 / 2} q^{-2}$. In this note we make a small change in Cohn's proof and get a much stronger result.

For $k \geq 1$, let $F(k)$ be the set of all real numbers $x$ such that $0 \leq x \leq$ 1 and the continued fraction for $x$ has no partial quotient greater than $k$. Let $F(0)=\varnothing$. Real numbers $x$ and $y$ are called equivalent if their continued fractions eventually coincide.

Theorem. Let $k \geq 1$ and let $x$ be a real irrational number not equivalent to an element of $F(k-1)$. Then there are infinitely many rational numbers plq such that

$$
|x-p / q|<1 /\left(k^{2}+4\right)^{1 / 2} q^{2} .
$$

The constant $\left(k^{2}+4\right)^{-1 / 2}$ is best possible.

Proof. Let $p_{n} / q_{n}$ denote the $n$th convergent of the continued fraction $\left[a_{0}, a_{1}, a_{2}, \cdots\right]$ of $x$. Following Cohn, we let $\theta_{n}=q_{n}\left|q_{n} x-p_{n}\right|$ and $\phi_{n}=$ $\min \left(\theta_{n-1}, \theta_{n}, \theta_{n+1}\right)$ and obtain

and so

$$
\frac{\theta_{n}}{q_{n}^{2}}+\frac{\theta_{n+1}}{q_{n+1}^{2}}=\left|x-\frac{p_{n}}{q_{n}}\right|+\left|x-\frac{p_{n+1}}{q_{n+1}}\right|=\frac{1}{q_{n} q_{n+1}},
$$

$$
\frac{q_{n+1}}{q_{n}}=\frac{1 \pm\left(1-4 \theta_{n} \theta_{n+1}\right)^{1 / 2}}{2 \theta_{n}} \text {. }
$$

Received by the editors July 10, 1973. AMS (MOS) subject classifications (1970). Primary $10 \mathrm{~F} 20$.

Key words and phrases. Hurwitz' theorem, continued fraction, diophantine approximation. 
Then

$$
\frac{1+\left(1-4 \theta_{n} \theta_{n+1}\right)^{1 / 2}}{2 \theta_{n}} \geq \frac{q_{n+1}}{q_{n}}=a_{n+1}+\frac{q_{n-1}}{q_{n}} \geq a_{n+1}+\frac{2 \theta_{n-1}}{1+\left(1-4 \theta_{n-1} \theta_{n}\right)^{1 / 2}},
$$

and

$$
2 \phi_{n} a_{n+1} \leq 2 \theta_{n} a_{n+1} \leq\left(1-4 \theta_{n} \theta_{n+1}\right)^{1 / 2}+\left(1-4 \theta_{n-1} \theta_{n}\right)^{1 / 2} \leq 2\left(1-4 \phi_{n}^{2}\right)^{1 / 2},
$$

or $\phi_{n} \leq\left(a_{n+1}^{2}+4\right)^{-1 / 2}$. If $\phi_{n}=\left(a_{n+1}^{2}+4\right)^{-1 / 2}$, then $\theta_{n}=\theta_{n+1}=\phi_{n}$. But, by (2), this is impossible. Therefore, $\phi_{n}<\left(a_{n+1}^{2}+4\right)^{-1 / 2}$ for all $n$.

If $x$ is not equivalent to an element of $F(k-1)$, then for infinitely many $n$ we have $a_{n+1} \geq k$, and so $\phi_{n}<\left(k^{2}+4\right)^{-1 / 2}$. This means that infinitely many convergents of the continued fraction for $x$ satisfy (1).

The continued fraction for $x=\left(\left(k^{2}+4\right)^{1 / 2}-k\right) / 2$ is $[0, k, k, k, \ldots]$, and so $x$ is not equivalent to an element of $F(k-1)$. If $A<\left(k^{2}+4\right)^{-1 / 2}$, it follows by a standard argument (see [2, Theorem 194] in the case $k=1$ ) that $|x-p / q|<A q^{-2}$ has only finitely many rational solutions $p / q$, and so the constant $\left(k^{2}+4\right)^{-1 / 2}$ is best possible.

Remark. For $k=1$, this theorem gives Hurwitz' theorem. The set $F(1)$ contains only the number $\left(5^{1 / 2}-1\right) / 2$, the golden mean, and so when $k=2$ the theorem gives the well-known result that, for any irrational number $x$ not equivalent to the golden mean, the inequality $|x-p / q|<8^{-1 / 2} q^{-2}$ has infinitely many rational solutions $p / q$.

\section{REFERENCES}

1. J. H. E. Cohn, Hurwitz' theorem, Proc. Amer. Math. Soc. 38 (1973), 436.

2. G. H. Hardy and E. M. Wright, An introduction to the theory of numbers, 4th ed., Oxford Univ. Press, London, 1960.

DEPARTMENT OF MATHEMATICS, SOUTHERN ILLINOIS UNIVERSITY, CARBONDALE, ILLINOIS 62901

Current address: School of Mathematics, The Institute for Advanced Study, Princeton, New Jersey 08540 